19 Revue d'histoire du XIXe siècle

Société d'histoire de la révolution de 1848 et des

révolutions du XIXe siècle

25 | 2002

Le temps et les historiens

\title{
Présent, passé, futur : la mort et le temps politique (1820-1830)
}

\section{Emmanuel Fureix}

\section{OpenEdition \\ Journals}

Édition électronique

URL : http://journals.openedition.org/rh19/433

DOI : $10.4000 /$ rh 19.433

ISSN : $1777-5329$

\section{Éditeur}

La Société de 1848

\section{Édition imprimée}

Date de publication : 1 décembre 2002

Pagination : 155-163

ISSN : 1265-1354

\section{Référence électronique}

Emmanuel Fureix, «Présent, passé, futur : la mort et le temps politique (1820-1830) », Revue d'histoire du XIXe siècle [En ligne], 25 | 2002, mis en ligne le 29 juin 2005, consulté le 19 avril 2019. URL : http:// journals.openedition.org/rh19/433; DOI : 10.4000/rh19.433

Ce document a été généré automatiquement le 19 avril 2019

Tous droits réservés 


\title{
Présent, passé, futur : la mort et le temps politique (1820-1830)
}

\author{
Emmanuel Fureix
}

1 Jamais depuis la Révolution le culte des morts n'avait suscité de telles mobilisations politiques. Sous la Restauration, les morts de princes, de "grands hommes", d'opposants voire d'émeutiers deviennent des événements politiques, où s'estompe le rite de passage individuel au profit d'une mise en scène publique ou d'une prise de parole collective ${ }^{1}$. Ces morts à usage politique sollicitent les trois dimensions temporelles, passé, présent, futur. Plus que tout autre événement, elles font s'entrecroiser un horizon d'attente et un champ d'expérience collectifs ${ }^{2}$, et à ce titre, informent sur les imaginaires du temps politique, dont les trois composantes sont rarement étudiées simultanément. Elles renvoient toujours, par delà le défunt, à une expérience commune, plus ou moins traumatique, et contribuent à ce titre à inscrire, parfois brutalement, le passé révolutionnaire dans le présent. Le discours ou le rituel funèbres agissent aussi sur le présent, subrepticement, sous la forme de l'avertissement ou de la contestation. Ces morts suscitent, enfin, des attentes d'avenir, plus ou moins crispées, capables de prolonger ici-bas l'héritage du défunt.

2 Le premier XIX siècle correspond à cet égard à un basculement du rapport passé/ présent/futur. Avec le sentiment tragique d'accélération du temps, s'est creusé l'écart entre les expériences acquises et le futur représenté ${ }^{3}$. Les grands événements funèbres de la Restauration sont les laboratoires privilégiés de ce processus. Ils font émerger deux cultures du temps, deux réponses possibles à cette dislocation du lien entre passé et futur, grossièrement superposables aux sensibilités ultra-royaliste et libérale. La première nie cette dislocation, fige le déroulement du temps dans une éternité fictive, dont le futur découlerait providentiellement d'un passé déjà écrit. La seconde fait du moment funèbre le point de passage vers la promesse de lendemains meilleurs, et l'un des signes de la perfectibilité de l'histoire humaine. Nous les étudierons essentiellement à partir de deux événements, la mort du duc de Berry (février 1820), et la mort du général libéral Foy 
(novembre 1825), et à l'aide de deux méthodes d'enquête : l'analyse des discours funèbres dans un cas, l'approche anthropologique dans l'autre, à partir du rituel des funérailles.

On connaît, jusqu'à la caricature, l'utopie réactionnaire des ultras ${ }^{4}$, ce vieux rêve de rétablissement de l'ordre immuable des choses, et d'effacement de la césure révolutionnaire. La mort violente du duc de Berry, héritier de la dynastie assassiné à la sortie de l'Opéra sous les coups de Louvel, la nuit du 13 au 14 février 1820, met à l'épreuve cette attente un peu désespérée du retour à l'ancien. La Révolution, par la main de Louvel, resurgit en pleine Restauration. Signe apocalyptique ? Dernière expiation d'un passé qui ne passe pas? L'événement funèbre, ici, offre le meilleur laboratoire qui soit des représentations ultra-royalistes du temps politique. Du décès du prince aux funérailles de Saint-Denis, le 14 mars 1820, les ultras, on le sait, font un retour en force, voient leurs exigences triompher, obtiennent le départ de Decazes et le dépôt de lois d'exception sur la presse et la liberté individuelle. Dans ce moment contre-révolutionnaire de la Restauration, que les libéraux comparèrent à la Terreur blanche de 1815, il est d'autant plus intéressant d'examiner, dans les discours ultras sur la mort du prince, comment la Providence permet d'intégrer un "accident politique" ${ }^{5}$ dans un temps monarchique "éternel".

Le présent, aux couleurs du régicide, est englouti dans la répétition du passé révolutionnaire. Le topos du "nouveau 21 janvier" prend forme dans la presse ultraroyaliste dès les lendemains du crime ${ }^{6}$. L'allusion au régicide ne relève pas de l'analogie historique, passage obligé de tout discours sur l'événement au XIX ${ }^{e}$ siècle, elle traduit cette étrange confusion des temps qui hante les ultras, l'angoisse de revivre une deuxième fois le drame central de la Révolution. Il faut prendre littéralement ces mots d'un royaliste intransigeant, début mars 1820, qui constate que "le passé vient de ressusciter tout entier dans le crime de Louvel" ${ }^{7}$.

5 Cette reviviscence de la Révolution, pour les ultras, est œuvre providentielle, signe d'un gouvernement divin. Elle accomplit une prophétie longtemps scandée. La prophétie, on le sait, est devenue depuis 1789 la clef de lecture contre-révolutionnaire de l'histoire contemporaine, guidée par une interprétation des Écritures, du jugement de Salomon à l'Apocalypse selon Saint-Jean ${ }^{8}$. Elle retrouve sous la Restauration un éclat particulier, en particulier auprès des ultras lecteurs du onzième entretien des Soirées de Saint-Petersbourg de Joseph de Maistre ${ }^{9}$, et témoins attentifs des oracles de Mademoiselle Lenormand ${ }^{10}$, ou du prophète Martin de Gallardon ${ }^{11}$. La prophétie permet, précisément, de sortir du temps, de se créer un substitut d'éternité ${ }^{12}$. En 1819-1820, les prophéties se multiplient dans le discours ultra, qui annoncent le triomphe de la révolution, voire du régicide. Le retour d'une partie des régicides bannis du territoire en $1815^{13}$, l'élection de l'abbé Grégoire à la Chambre des députés, assimilé à un "régicide", les soulèvements révolutionnaires en Espagne, les dénonciations libérales des cérémonies expiatoires du 21 janvier ${ }^{14}$, font pressentir, dans les mois et semaines qui précèdent l'assassinat du duc, l'imminence de la catastrophe ${ }^{15}$.

6 Au lendemain du 13 février, la catastrophe est donc lue comme prophétie réalisée. Une brochure publiée anonymement recense rétrospectivement 43 "pressentiments, rêves, visions, apparitions et singularités" annonciateurs de la mort du prince ${ }^{16}$. Les anecdotes sont peuplées de signes surnaturels, et reposent sur les croyances populaires, surtout féminines, en la clairvoyance des rêves prémonitoires ${ }^{17}$. Le propos sous-jacent en est proprement politique: le régicide répond à une effroyable logique, prévisible et observable, l'affaiblissement du principe monarchique, autorisé par une dérive libérale 
du régime, incarnée par Decazes. Dans le même esprit, la mort du duc redonne un sursaut d'actualité aux avertissements donnés depuis janvier 1816 par le prophète royal Martin, laboureur beauceron, et relayés par des cercles dévots et une camarilla ultra. Les visions de l'oracle-paysan, en effet, s'étaient poursuivies après son entrevue avec le roi le 2 avril 1816 et, depuis 1819, se faisaient l'écho des sombres menaces qui pesaient sur la Restauration. Leur confirmation par la mort du prince-héritier de la dynastie redonne sens aux voix de la Providence, et tort à la modération de Louis XVIII : "Rougissons d'être français, s'exclame le très royaliste marquis de Sailly. Quel crime affreux vient d'être commis [...]. Voilà la suite de tous les malheurs prédits par Martin, s'il [le roi] ne profitait pas des avertissements de l'ange" ${ }^{18}$.

7 Cette menace du passé révolutionnaire sur le présent doit, précisément, être conjurée. Durant la cérémonie funèbre, des allusions à la Révolution proposent une réécriture du passé, à rebours, au profit des vaincus. L'oraison funèbre très virulente prononcée à Saint-Denis par le futur archevêque de Paris Hyacinthe de Quélen glorifie les hauts-faits de l'armée de Condé ${ }^{19}$, à la façon de Chateaubriand dans son éloge du prince ${ }^{20}$. Cette réécriture de l'histoire s'accompagne d'un appel à agir sur le présent, pour garantir l'avenir des Bourbons. Agir pour mieux extirper les "fausses doctrines", l'athéisme et le libéralisme porté par "mille plumes empoisonnées" ${ }^{21}$. Ce travail sur le présent suppose l'achèvement des exercices d'expiation et de pénitence collectives, auxquels invitent toutes les oraisons funèbres prononcées ${ }^{22}$.

8 Une expiation d'autant plus nécessaire que les représentations de l'avenir semblent floues. L'interprétation sacrificielle de la mort du prince invite, certes, à l'optimisme : la réversibilité des fautes théorisée par Joseph de Maistre fait du sang du juste l'instrument du salut collectif. Le même Joseph de Maistre croit discerner, à la nouvelle de la mort du duc de Berry, la fin du temps de l'expiation: "Nous chantons bien à l'église Felix culpa! pour le plus grand de tous les crimes, puisqu'il a perdu le genre humain. Pourquoi ne nous permettrions-nous pas la même exclamation en voyant dans l'avenir tout ce que doit produire cette grande mort toute vitale et vivifiante ? [...] N'en doutez pas, Monsieur le Vicomte, nous venons de voir la fin des expiations. Le Régent même et Louis XV ne doivent plus rien, et la Maison de Bourbon a reçu l'absolution" ${ }^{23}$.

9 Prolongeant la lecture de la Restauration comme série de miracles, les oraisons funèbres interprètent la lenteur de l'agonie du prince comme une grâce miraculaire ${ }^{24}$ : elle lui aurait permis d'accomplir symboliquement ses vertus, notamment par le pardon accordé à Louvel. Cette grâce en annoncerait d'autres, et le salut de la monarchie et de la religion passerait par l'accomplissement d'un "prodige en faveur de la postérité de saint Louis" ${ }^{25}$, l'enfant porté par la duchesse de Berry.

10 Mais tous les ultras ne s'accordent pas sur cette représentation apaisée de l'avenir. Le sang versé ne prémunit pas des craintes du pressentiment. Villèle, futur président du conseil ultra, écrit à sa femme le 18 février : “Je puis t'assurer d'après notre expérience que le temps ne fait que l' [la douleur] accroître par le sentiment des dangers que cet attentat a rendus plus évidents. On se voit au fond de l'abîme et l'on ne sait comment en sortir" ${ }^{26}$.

11 L'interprétation prophétique des dernières paroles du défunt --"O France... malheureuse patrie" ${ }^{27--}$, grand classique des récits de morts, annonce pour certains "l'éruption prochaine du volcan révolutionnaire" ${ }^{28}$. Un missionnaire use d'un ton apocalyptique pour prédire "une ruine générale et prochaine" de l"univers entier" ${ }^{29}$. C'est que la mort violente, pour être expiatoire, n'en révèle pas moins un arrêt de la Providence, un 
avertissement donné au monarque. La mort est la délivrance d'un "secret" ${ }^{30}$, que seul un raidissement du régime peut, éventuellement, rendre salvateur. La grande et belle mort du duc de Berry, dont chacune des séquences épouse si bien les formes du drame romantique ${ }^{31}$, donne aussi à voir un temps politique très proche du temps romantique, fait de mémoire affective, d'angoisse du futur, et d'un sentiment de perte ${ }^{32}$.

La mort de personnalités d'opposition devint, à partir des années 1820, l'occasion de démonstrations d'opinion. S'invente dans le Paris de la Restauration un nouveau rituel politique, proche à certains égards de la manifestation, confinant même à la révolte ou à l'insurrection sous la Monarchie de Juillet ${ }^{33}$. Ces enterrements d'opposition donnent à voir une autre perception du temps politique, un autre mode de liaison passé/présent/ futur, d'où est exclue la providence pour faire toute sa place à la volonté collective. Ce discours sur le temps s'y lit davantage dans le moment des funérailles que dans les réactions immédiates à la mort. Le cortège, Mona Ozouf l'a amplement souligné à propos de la fête révolutionnaire, travaille, façonne, ordonne le temps ${ }^{34}$. Les cortèges funèbres des années 1820, par leur ordonnancement, leurs gestes, leurs mots prononcés, expriment une politique du temps, que nous qualifierons de libérale, sans que ses auteurs puissent être toujours clairement identifiés. Nous nous appuierons en particulier sur les funérailles du général Foy, le 30 novembre 1825.

Le républicain Armand Marrast, qui décrit dès 1834 le nouveau rituel des "funérailles révolutionnaires" apparu sous la Restauration, voit dans cette politique du temps un art de l'avertissement, où le passé vient télescoper le présent pour produire l'événement: "Aujourd'hui donc, écrit-il, [...] au lieu de ces cérémonies graves et tristes, et de ces prières mêlées d'espérance et de crainte, dont le christianisme accompagnait le dernier voyage, nous avons des obsèques bruyantes, agitées ; où les souvenirs ont plus de part que la douleur, où l'on menace le présent en enterrant le passé" ${ }^{35}$.

14 Les souvenirs, de fait, s'immiscent dans le cortège pour célébrer audacieusement l'aventure révolutionnaire et impériale, et renvoient à des interdits de représentation dans le présent. L'épée et les épaulettes du général Foy, posées sur le cercueil, saluées et baisées par la foule, évoquent aux spectateurs Jemmapes et Waterloo, autant de références taboues sous la Restauration, mais relayées par la presse et les brochures libérales du moment. Les couronnes civiques déposées sur le cercueil soulignent également la continuité avec une pratique révolutionnaire. Le phénomène se prolonge au cours des années 1820-1830, et en juin 1832 s'observe encore une forte mobilisation patriotique autour du passé révolutionnaire et impérial du général Lamarque : brochures et presse, à la veille des funérailles, égrènent les campagnes du volontaire de 1792 et du pacificateur de la Vendée, tandis que le cortège joue avec les lieux de mémoire de la Révolution et de l'Empire. Un premier détournement du cortège autour de la colonne Vendôme est suivi d'une station place de la Bastille, et d'une tentative de détournement vers le Panthéon. Le temps funèbre des libéraux et des républicains prétend récapituler le passé collectif autour de ses références les plus tues.

15 Sans contradiction, les funérailles libérales sont aussi tendues vers une célébration de l'avenir, occultant quelque peu le passage du défunt vers l'au-delà et son agrégation au monde des morts. Les allusions à l'au-delà, dans des cérémonies faiblement chrétiennes -funérailles du général Foy-- ou clairement civiles --funérailles du député Manuel en août 1827-- sont estompées par les attentes d'avenir ici-bas, dont la foule se fait le portevoix. Les funérailles libérales --puis républicaines sous la Monarchie de Juillet-- sont des moments d'espoir collectif autant que d'affliction, des ovations autant que des adieux. 
Indice de ces attentes d'avenir, la jeunesse est placée au cœur d'un rituel dont elle n'a pas habituellement le contrôle. C'est elle qui invente le rituel politique des funérailles d'opposition, à l'occasion de la mort de l'étudiant en droit Nicolas Lallemand en juin 1820 : 6000 étudiants suivent le cercueil de leur camarade, crient Vive la charte! dans l'enceinte du cimetière et prolongent le cortège par une promenade civique sur les boulevards. C'est elle, en l'occurrence des étudiants et des commis, qui s'empare du cercueil du général Foy, et le porte à bras, en contradiction avec les usages funéraires ${ }^{36}$. Elle est aussi au premier plan lors des troubles observés au cours des funérailles de La Rochefoucauld-Liancourt et de Manuel en 1827, suscités par le même port à bras du cercueil. Ce dernier exprimait la conscience d'un héritage à prolonger autant qu'un sentiment de reconnaissance au grand homme.

De même, les enfants du défunt prennent une part significative au cortège, placés près du corbillard, en l'absence de la veuve. Il ne s'agit pas seulement de catalyser l'émotion et renforcer le sentiment du sublime, comme l'avaient pratiqué les révolutionnaires. Les enfants du défunt s'identifient avec la nation opposante, qui survit à la mort du grand homme, et sont mis en scène comme tels dans le cortège. Lors des funérailles du général Foy, ses trois fils mènent le deuil, deux d'entre eux sont tenus par la main par CasimirPérier, autre opposant notoire aux ultras. L'inhumation se conclut également par l'adoption des enfants du général par la nation réunie en corps, au moyen d'une souscription publique. Plus tard, en 1832, le fils du général Lamarque suit, derrière le char funèbre, des invalides qui portaient les insignes du défunt, près de son cheval de bataille. Passé et avenir sont symboliquement réunis dans le cortège. Au compartimentage des temps, caractéristique des fêtes de la Révolution ${ }^{37}$, s'oppose ici un mélange des âges, propre à susciter le sentiment d'une immortalité politique et d'une continuité de l'histoire. Cette immortalité politique pouvait également s'exprimer dans des cris de reconnaissance qui confondaient dans leur hommage le défunt et les ténors libéraux qui lui survivaient : la foule scande ainsi, lors des funérailles de Manuel, "Honneur à Manuel! honneur à Lafitte! à Lafayette! à Béranger !" ${ }^{38}$.

De fait, les funérailles libérales de la Restauration visaient à préparer l'avenir, à diffuser une pédagogie politique, à annoncer des lendemains meilleurs. Dans ces circonstances, le passé du défunt prenait un sens futur : inaccompli, identifié avec celui des "vaincus" de l'histoire, il s'actualisait dans la promesse d'une action à venir, située dans un futur indéterminé. C'est dans les derniers instants précédant l'inhumation que se formulait cette promesse, par les éloges funèbres ou les serments prononcés sur la tombe. Lors des funérailles du général Foy, témoigne un contemporain, "un serment tacite a été prononcé sur la dépouille illustre de l'homme de la patrie. Sa mort a imprimé au corps social un mouvement irrésistible d'ordre et d'indépendance, de force et de sagesse" ${ }^{39}$. Les funérailles libérales, reposant sur la religion de l'exemplarité, transmettaient un héritage pour le futur. En mars 1827, lors des obsèques de Stanislas de Girardin, député libéral de Rouen, un des orateurs conclut son discours au Père-Lachaise "en jurant [...] de défendre comme lui les libertés publiques jusqu'à son dernier soupir" ${ }^{40}$. Les enterrements figuraient, paradoxalement, autant de commencements, dans lesquels Armand Marrast voyait les "préludes de cette pensée d'émancipation générale qui dormait encore au fond de la conscience publique" ${ }^{41}$, balbutiements d'une contestation générale de la Restauration.

L'avenir ainsi projeté était actualisé dans le présent, par des allusions au contexte politique contemporain. Cette configuration de l'avenir dans le présent était moins visible 
dans le cortège que dans les représentations des funérailles, dans les jours suivant l'événement. La foule, en effet, poussait peu de cris séditieux, se contentant de paroles trop vagues pour exprimer des revendications précises ${ }^{42}$; en ce sens les funérailles libérales se distinguaient nettement de la manifestation moderne. Elles visaient cependant à dire un état de l'opinion et un rapport de force, explicités dans la mise en récit des cortèges. Des brochures diffusées et parfois censurées ${ }^{43}$, des articles de presse également censurés, formulaient aposteriori le sens politique de ces mobilisations funèbres. Ainsi les funérailles du général Foy, dans le contexte de procès de presse visant le Constitutionnel et le Courrier français, furent-elles érigées par les libéraux en lutte, dans le présent et l'avenir, pour la liberté d'expression. D'autres en firent un "vote pour la charte" ${ }^{44}$ contre un retour à l'ordre ancien, quelques mois après l'adoption de la loi sur le sacrilège ${ }^{45}$. Ce travail de représentation peut s'accompagner d'un appel immédiat à l'action, notamment à l'issue des funérailles de Manuel où une brochure relatant l'événement se conclut par un appel électoral signé de la société Aide-toi, le ciel t'aidera, qui invite à voter pour les candidats libéraux, héritiers politiques du défunt ${ }^{46}$.

Sous la Monarchie de Juillet, cette continuité de l'histoire dessinée par les funérailles libérales tend à se briser. Dès les funérailles de Benjamin Constant, en décembre 1830, plus encore à partir de celles du général Lamarque en juin 1832, l'enterrement d'opposition devient une geste de menace, un face-à-face avec le pouvoir dont l'issue serait suspendue. L'avenir meilleur attendu sur la durée par les libéraux, est plaqué sur le présent par les républicains. Indice rituel de cette métamorphose des temps, Blanqui en décembre 1830, les Amis du Peuple en juin 1832, lors des obsèques de Lamarque, la Société des Droits de l'Homme en février 1834, lors des obsèques de Dulong, appellent leurs amis républicains à manifester en armes. Les républicains voient dans les funérailles une de ces fractures où le temps peut s'arrêter pour engendrer du nouveau, où l'émotion funèbre peut dériver sur l'insurrection. Espérance pour partie brisée par l'échec du cloître Saint-Merry, mais qui durablement fit des funérailles révolutionnaires un "état d'exception" ${ }^{47}$.

Emmanuel Fureix est doctorant

21 à l'Université Paris 1-Panthéon-Sorbonn

\section{NOTES}

1.. Notre thèse, en cours, porte sur les mises en scène politiques de la mort sous les monarchies censitaires (1814-1835).

2.. Reinhart KOSELLECK, “Champ d'expérience et horizon d'attente : deux catégories historiques”, dans Reinhart KOSELLECK, Le futur passé. Contribution à la sémantique des temps historiques, Paris, Éditions de l'École des hautes études en sciences sociales, 1990, pp. 307-330.

3.. Ce que Lamartine, dans son Histoire de la Restauration, dit admirablement : "Il n'y a plus d'histoire contemporaine. Les jours d'hier semblent déjà enfoncés bien loin dans l'ombre du passé. Les perspectives reculent par la grandeur et la multitude des choses qui 
s'interposent entre l'œil et la mémoire" ; voir Alphonse de LAMARTINE, Histoire de la Restauration, Paris, Éditions Lecou, 1851, tome 1, p. 1.

4.. Car on l'a trop souvent identifiée avec le projet politique de la Restauration tout entière.

5.. Le Drapeau Blanc, 17 février 1820.

6.. Chateaubriand évoque ainsi la "grande victime du nouveau 21 janvier" ; voir FrançoisRené de CHATEAUBRIAND, “lettre parisienne”, dans Le Conservateur, 18 février 1820.

7.. A.-J.-C. SAINT-PROSPER, La France royaliste aux mânes de Mgr le duc de Berry, Paris, Pichard et Le Normand, 1820.

8. Ces interprétations resurgissent sous la Restauration, suscitent débat et reçoivent un écho dans l'Ami de la Religion et du Roi. Citons notamment l'Explication de l'allégorie prophétique contenue dans le jugement de Salomon, par l'abbé Cadart, Épernay, 1816, 94 p., et Les Précurseurs de l'Antechrist, histoire prophétique des plus fameux impies qui ont paru depuis l'établissement de l'Église jusqu'à nos jours, ou la révolution française prédite par saint Jean l'évangéliste... Septième édition, Lyon, Rusand, 1822.

9.. Nicole Edelman souligne, par ailleurs, la fascination des émigrés pour le magnétisme et le somnambulisme visionnaire ; voir Nicole EDELMAN, Voyantes, guérisseuses et visionnaires en France. 1785-1914, Paris, Éditions Albin Michel, 1995, p. 60.

10.. Modèle de la voyante pour le $\mathrm{XIX}^{\mathrm{e}}$ siècle, elle se rallie avec passion à la cause des Bourbons en 1815.

11.. Voir Philippe BOUTRY et Jacques NASSIF, Martin l'archange, Paris, Éditions Gallimard, 1985.

12.."L'homme, en essayant, à toutes les époques et dans tous les lieux, de pénétrer dans l'avenir, déclare qu'il n'est pas fait pour le temps ; car le temps est quelque chose qui ne demande qu'à finir" ; Joseph de MAISTRE, Les Soirées de Saint-Petersbourg, Onzième entretien, dans Euvres complètes, Lyon, Librairie générale catholique classique, 1884, tome 5, p. 235.

13.. Par ordonnance royale, pour les plus âgés et malades d'entre eux. En mai 1819, une série de pétitions adressées aux chambres demandent le retour de tous les bannis.

14.. Les Lettres normandes publient en 1820, sous la plume de Léon Thiessé, un article virulent contre le ressassement expiatoire du 21 janvier, qui vaut à l'auteur et à l'éditeur un procès en cour d'assises pour atteinte à l'inviolabilité de la personne royale.

15.. À titre d'exemple, citons Chateaubriand, qui dans une lettre adressée à Joseph de Maistre le 25 janvier 1820, écrit : "Pour moi, je crois la France perdue, et par conséquent l'Europe ; non qu'il ne nous reste encore des moyens de nous sauver, mais on ne les prendra pas" ; voir François-René de CHATEAUBRIAND, Correspondance générale, Paris, Éditions Gallimard, 1980-1986, tome 3, p. 230. Parmi les témoignages ironiques sur ce prophétisme apocalytpique, citons une caricature libérale de juillet 1819 intitulée "le désespoir des ultras, ou la comète de 1819". Elle représente des ultras désespérés de voir des bonnets rouges sur la comète ; Lithographie de Plancher, Collection de Vinck, tome 80, Bibliothèque nationale, Cabinet de Estampes, M 10498.

16.. Pressentimens, rêves, visions, apparitions et singularités qui ont précédé la mort de S.A.R Mgr le duc de Berry ; recueillis et publiés par Madame la marquise de $W^{* * *}$, Paris, Moreau, 1820. 17.. Voir Yannick RIPA, “Mort du rêve prémonitoire ?”, dans Yannick RIPA, Histoire du rêve. Regard sur l'imaginaire des Français au XIX siècle, Paris, Olivier Orban, 1988, pp. 93-102. La majorité des rêves rapportés dans la brochure sont féminins.

18. Lettre du marquis de Sailly citée par Ernest DAUDET, L'ambassade du duc Decazes en Angleterre (1820-1821), Paris, Librairie Plon, 1910, p. 285. 
19.."Il servit ensuite sous les yeux d'un prince qui devait devenir son Roi et le nôtre, dans cette armée qui ne connut, il faut avoir le courage de le dire, ni défections, ni défaites, commandée qu'elle était par trois Condé" ; voir M. de QUÉLEN, Oraison funèbre de Son Altesse Royale monseigneur le duc de Berry, prononcée dans l'église royale de Saint-Denis, par M. de Quélen, coadjuteur de monseigneur l'archevêque de Paris, Marseille, Dubié, s.d., pp. 3-4.

20.. François-René de CHATEAUBRIAND, Mémoires, lettres et pièces authentiques touchant la vie et la mort de Son Altesse Royale Monseigneur Charles-Ferdinand d'Artois, Fils de France, duc de Berry, Paris, Imprimerie de Le Normant, 1820.

21.. M. de QUÉLEN, Oraison funèbre ..., ouv. cité, p. 7.

22.. Que nous ne pouvons citer, dans les limites de l'article.

23.. Joseph de MAISTRE, "Lettre de Joseph de Maistre au vicomte de Bonald, 25 mars 1820", dans Joseph de MAISTRE, Correspondance. 1815-1821, dans Joseph de MAISTRE, CEuvres Complètes. Nouvelle édition, Lyon, Vitte et Perrussel, 1886, tome 13, pp. 213-214.

24.."J'aperçois d'abord une grâce qui renferme toutes les autres, une grâce que je devrais peut-être appeler un miracle, car vous le savez, messieurs, et l'unanime opinion des plus savants maîtres nous autorise à le dire, le Prince, après cet effroyable coup, ne pouvait conserver quelques instants de vie sans que les lois de la nature ne parussent manifestement suspendues" ; voir Oraison funèbre de S. A. R. monseigneur le duc de Berry, par l'abbé $D^{* * *}$, Paris, Dentu, s.d., p. 18.

25.. Mandement de Son Éminence Monseigneur le Cardinal Archevêque de Paris, qui ordonne qu'il sot célébré un service solennel pour le repos de l'âme de Son Altesse Royale Mgr le Duc de Berry, Paris, Le Clère, 1820, p. 12.

26.. VILLÈLE, Mémoires et correspondance du comte de Villèle, Paris, Librairie académique Perrin, 1888-1890, tome II, p. 341.

27.. J.-B.-A. HAPDÉ, Relation historique des événements funèbres de la nuit du 13 février 1820, Paris, Dentu, 1820, p. 27.

28.. L'Anniversaire du 13 février 1820, Paris, imp. de Guiraudet, 1823, p. 11.

29.. Abbé ENFANTIN, Oraison funèbre de son Altesse Royale monseigneur Charles-Ferdinand d'Artois, fils de France, duc de Berry, improvisée par M. l'abbé Enfantin, missionnaire, dans l'église de Saint-Roch à Paris, Valence, Montal, 1820, p. 21.

30.. Oraison funèbre de S.A. R. monseigneur le duc de Berry, par l'abbé $D^{* * *}$, ouv. cité, p. 15.

31.. Christine MARCANDIER-COLARD, Crimes de sang et scènes capitales. Essai sur l'esthétique romantique de la violence, Paris, Presses universitaires de France, 1998, 298 p.

32.. Nous renvoyons ici aux belles analyses de Georges POULET, Études sur le temps humain/1, Paris, Éditions Pocket, 1989, Introduction.

33.. Nous renvoyons à nos deux articles : Emmanuel FUREIX, "De l'hommage funèbre à la prise de parole : l'enterrement du général Foy (novembre 1825)", dans Sociétés et Représentations, juin-septembre 2001, pp. 177-204 ; et Emmanuel FUREIX, "Un rituel d'opposition sous la Restauration : les funérailles libérales à Paris (1820-1830)", dans Genèses. Histoire, sciences sociales, $\mathrm{n}^{\circ}$ 46, mars 2002, pp. 77-100.

34.. Mona OZOUF, La Fête révolutionnaire. 1789-1799, Folio, Paris, Éditions Gallimard, 1993 (1 ère édition 1976), pp. 260 et suivantes.

35.. Armand MARRAST, "Les funérailles révolutionnaires", dans Paris révolutionnaire, Paris, Éditions Pagnerre, 1848 (1 ${ }^{\text {ère }}$ édition 1834), p. 5.

36.. Le transport du cercueil sur un char funèbre était obligatoire.

37.. Mona Ozouf souligne cette séparation des âges dans les célébrations de la morts du roi et les fêtes consacrées à la jeunesse, aux Époux et aux vieillards : Mona OZOUF, La Fête révolutionnaire..., ouv. cité, p. 311. 
38. M. MIGNET, Relation historique des obsèques de M. Manuel, ancien député de la Vendée, Paris, Imprimerie de Gaultier-Laguionie, 1827, p. 11.

39. Journée du 30 septembre 1825, ou récit des derniers moments et des funérailles du général Foy, Paris, Mongie aîné, 1825, p. 8.

40. Voir Le Constitutionnel, 2 mars 1827.

41.. Armand MARRAST, "Les funérailles révolutionnaires", ouv. cité, p. 48.

42. Lors des funérailles du général Foy, ces cris sont plutôt des hommages de reconnaissance au défunt : "Vive le général Foy ! Honneur et gloire au général Foy ! Au défenseur des libertés publiques ! Il était notre défenseur ! C'était l'homme de la patrie..." 43. Notamment en 1827 après les funérailles de Manuel.

44.. Dans Le journal des débats, 3 décembre 1825.

45.. Adoptée le 30 avril 1825.

46.. Ainsi peut-on lire dans M. MIGNET, Relation historique... ouv. cité, p. 30 : "Vous auriez sans doute appelé à vous représenter dans les élections qui vont s'ouvrir, le digne citoyen que l'on vient de perdre ; songez que la meilleure manière d'honorer sa mémoire et de venger l'outrage qu'on lui a fait, est de faire constater vos droits pour changer la majorité qui l'en exclut. Souvenez-vous de notre devise : Aide-toi, le ciel t'aidera".

47. Expression que nous empruntons à Walter BENJAMIN, "Sur le concept d'histoire, VIII”, dans Euvres, Folio, Paris, Éditions Gallimard, année, tome 3, pp. 433. 